\title{
ORDER OF NORMAL APPROXIMATION FOR RANK TEST STATISTICS DISTRIBUTION
}

\author{
By Jana Jurečková and Madan L. Puri ${ }^{1}$ \\ Charles University and Indiana University
}

0. Summary. Under suitable assumptions, it is established that the rate of convergence of the cdf (cumulative distribution function) of the simple linear rank statistics

$$
S_{N}=\sum_{i=1}^{N} C_{N i} \varphi\left(\frac{R_{N i}}{N+1}\right)
$$

to the normal one is $O\left(N^{-\frac{1}{2}+\delta}\right)$ for any $\delta>0$. Here $C_{N 1}, \cdots, C_{N N}$ are known constants, $R_{N 1}, \ldots, R_{N N}$ are the ranks of independent observations $X_{N 1}, \ldots$, $X_{N N}$, and $\varphi$ is a score generating function defined in Section 1.

1. Introduction. Let $X_{N i}, i=1, \ldots, N$ be independent rvs distributed according to the cdf $F_{i}(x)=F\left(x-\Delta d_{N i}\right), i=1, \cdots, N$. We assumed that $F(x)$ is absolutely continuous having the density function $f(x)$ whose derivative $f^{\prime}(x)$ exists. Furthermore, $F(x)$ is assumed to have the finite Fisher information, that is,

$$
I(f)=\int_{-\infty}^{\infty}\left[f^{\prime}(x) / f(x)\right]^{2} f(x) d x<\infty .
$$

$\Delta$ is an unknown parameter, and $d_{N i}, i=1, \cdots, N$ are known constants. Let $R_{N i}$ be the rank of $X_{N i}$ among $X_{N 1}, \cdots, X_{N N}$. Setting $u(x)=1$ if $x \geqq 0$, and $u(x)=0$ otherwise, we can write

$$
R_{N i}=\sum_{j=1}^{N} u\left(X_{N i}-X_{N j}\right), \quad i=1, \ldots, N .
$$

Consider now the simple linear rank statistics

$$
S_{N}=\sum_{i=1}^{N} C_{N i} a_{N}\left(R_{N i}\right)
$$

where $C_{N 1}, \ldots, C_{N N}$ are known constants, and $a_{N}(i), i=1, \ldots, N$ are "scores" generated by a function $\varphi(t)$ in the following manner:

$$
a_{N}(i)=\varphi\left(\frac{i}{N+1}\right), \quad 1 \leqq i \leqq N .
$$

Statistics of the type (1.3) play an important role in the theory of nonparametric inference. For example, in the two sample problem where $F_{1}=\cdots=F_{m} \equiv F$, and

$$
F_{m+1}=\cdots=F_{N} \equiv G,
$$

Received March 26, 1974; revised October 1, 1974.

${ }^{1}$ Work supported by the Air Force Office of Scientific Research, AFSC, USAF, under Grant No. AFOSR 71-2009. Reproduction in whole or in part is permitted for any purpose of the U.S. Government. Part of this work was done while the author was the recipient of a Senior U.S. Scientist Award from the Alexander-von-Humboldt-Foundation.

AMS 1970 subject classifications. Primary 62E20; Secondary 60F05, 60F99.

Key words and phrases. Linear rank statistics, score generating function, rate of convergence. 
for testing the hypothesis $H_{0}: F \equiv G$, many rank tests are based on the statistic

$$
S_{N}^{\prime}=\sum_{i=1}^{m} a_{N}\left(R_{N i}\right)
$$

which is a special case of (1.3) when $C_{N 1}=\cdots=C_{N m}=1$ and $C_{N m+1}=\cdots=$ $C_{N N}=0$. It is well known (see e.g., Capon (1961)) that the statistics of the form (1.3) for different score functions yield locally most powerful rank tests. Under suitable assumptions on the $C$ 's and the score generating function $\varphi$, Hájek (1962) [see also Hájek-Šidák (1967)] established the asymptotic normality of $S_{N}$. However, the problem of determining the rate of convergence of the cdf of $S_{N}$ to the limiting normal distribution has remained open. This problem is investigated in this paper for the case $\Delta=0$ as well as for $\Delta \neq 0$. In both cases, the rate of convergence is proved to be $O\left(N^{-\frac{1}{2}+\delta}\right)$ for $\delta>0$. For the case $\Delta=0$, the result is valid for the $\varphi$ functions having the bounded first derivative, and for the case $\Delta \neq 0$, it is necessary to assume the boundedness of the fourth derivative of $\varphi$.

Throughout the paper, we shall make the following assumptions on $C$ 's and $d$ 's.

$$
\begin{gathered}
\sum_{i=1}^{N} C_{N i}=\sum_{i=1}^{N} d_{N i}=0, \quad \sum_{i=1}^{N} C_{N i}^{2}=\sum_{i=1}^{N} d_{N i}^{2}=1, \\
\max _{1 \leqq i \leqq N} C_{N i}^{2}=O\left(N^{-1} \log N\right), \quad \max _{1 \leqq i \leqq N} d_{N i}^{2}=O\left(N^{-1} \log N\right) .
\end{gathered}
$$

It may be noted that the assumption (1.5) can be made without any loss of generality. Furthermore, it may be noted [cf. Hájek-Šidák (1967)] that if $\varphi$ is the difference of two non-decreasing, square integrable functions in $(0,1)$, then $S_{N}$ has asymptotically $\eta\left(0, \sigma^{2}\right)$ distribution under $\Delta=0$, and $\eta\left(E S_{N}, \sigma^{2}\right)$ or

$$
\eta\left(\Delta \sum_{i=1}^{N} C_{N i} d_{N i} \int_{0}^{1} \varphi(t) \varphi(t, f) d t, \sigma^{2}\right)
$$

distribution under $\Delta \neq 0$. Here

$$
\sigma^{2}=\int_{0}^{1}(\varphi(t)-\bar{\varphi})^{2} d t, \quad \bar{\varphi}=\int_{0}^{1} \varphi(t) d t, \quad \varphi(t, f)=\frac{-f^{\prime}\left(F^{-1}(t)\right)}{f\left(F^{-1}(t)\right)}
$$

and $\eta\left(\xi, \sigma^{2}\right)$ stands for the normal distribution with mean $\xi$ and variance $\sigma^{2}$.

2. Rate of convergence for $\Delta=0$. The main result of this section is the following theorem.

THEOREM 2.1. Let $\Delta=0$ and the first derivative of $\varphi(t)$ exist and be bounded in $(0,1)$. Then, under the assumptions of Section 1 , corresponding to any $\delta>0$, there exists a constant $A(\delta)>0$, and a positive integer $N_{\delta}$ such that for all $N>N_{\delta}$,

$$
\sup _{-\infty<x<\infty}\left|F_{N}(x)-\Phi(x)\right| \leqq A(\delta) N^{\left(-\frac{1}{2}+\delta\right)}
$$

where $F_{N}(x)$ is the cdf of $\sigma^{-1} S_{N}$ and $\Phi(x)$ is the standard normal cdf.

The proof of this theorem is based on the following two lemmas, the second of which is a consequence of Theorem 6, Chapter 5 of Petrov (1972).

LEMMA 2.1. Under the assumptions of Theorem 2.1, corresponding to any positive integer $k$, where $2 k+1<N$, there exists a constant $B(k)>0$ and a positive integer $N_{k}$ such that for all $N>N_{k}$,

$$
E\left(S_{N}-T_{N}\right)^{2 k} \leqq B(k) N^{-k}
$$


where

$$
T_{N}=\sum_{i=1}^{N} C_{i} \varphi\left(F\left(X_{i}\right)\right)
$$

LeMma 2.2. Under assumptions of Section 2 and Theorem 2.1, for any positive integer $N$,

$$
\sup _{-\infty<x<\infty}\left|F_{N}{ }^{*}(x)-\Phi(x)\right| \leqq A \int_{0}^{1}|\varphi(t)-\ddot{\varphi}|^{3} d t \cdot \sum_{i=1}^{N}\left|C_{N i}\right|^{3}
$$

where $A>0$ is a constant independent of $N$, and $F_{N}{ }^{*}$ is the $\operatorname{cdf}$ of $\sigma^{-1} T_{N}$ under $\Delta=0$.

In what follows, we shall suppress the subscript $N$ in $C_{N i}, d_{N i}, R_{N i}$, etc. whenever there is no confusion.

Proof of Lemma 2.1. Set $U_{i}=F\left(X_{i}\right), i=1, \cdots, N$. Denoting $Y_{i}=a_{N}\left(R_{i}\right)-$ $\varphi\left(U_{i}\right), i=1, \cdots, N$, we get

$$
\begin{aligned}
E\left[\left(S_{N}-T_{N}\right)^{2 k}\right] & =E\left\{\left(\sum_{i=1}^{N} c_{i} Y_{i}\right)^{2 k}\right\} \\
& =\sum \frac{(2 k) !}{p_{1} ! \cdots p_{N} !} c_{1}^{p_{1}} \cdots c_{N}{ }^{p_{N}} E\left(\prod_{i=1}^{N} Y_{i}{ }^{p_{i}}\right)
\end{aligned}
$$

where the sum extends over the set $A$ of vectors $\left(p_{1}, \cdots, p_{N}\right)$ of integers such that $0 \leqq p_{i} \leqq 2 k, i=1, \cdots, N, \sum_{i=1}^{N} p_{i}=2 k$.

Each point of $A$ could have at most $2 k$ positive components. Noting this fact, we may decompose $A$ into $2 k$ disjoint parts such that the $j$ th part consists of those points which have just $j$ positive components. Thus we may rewrite $(2.5)$ as

$$
\begin{aligned}
& E\left[\left(S_{N}-T_{N}\right)^{2 k}\right]=\sum_{i=1}^{N} c_{i}^{2 k} E Y_{i}^{2 k}+\cdots \\
& +\sum_{1 \leqq p_{1}, \cdots, p_{m}<2 k, p_{1}+\cdots+p_{m}=2 k} \frac{(2 k) !}{p_{1} ! \cdots p^{m} !} \\
& \times \sum_{i_{1}, \cdots, i_{m}=1, \text { different }}^{N} c_{i_{1}}^{p_{1}} \cdots c_{i_{m}}^{p_{m}} E\left(Y_{i_{1}}^{p_{1}} \cdots Y_{i_{m}}^{p_{m}}\right)+\cdots \\
& +\sum_{i_{1}, \cdots, 2 k}^{N}=1, \text { different } c_{i_{1}} \cdots c_{i_{2 k}} E\left(Y_{i_{1}} \cdots Y_{i_{2 k}}\right) \text {. }
\end{aligned}
$$

In view of (1.5) and (1.6), it follows that

$$
\left|\sum_{i_{1}, \cdots, i_{m}=1, \text { different }}^{N} c_{i_{1}}^{p_{1}} \cdots c_{i_{m}}^{p_{m}}\right| \leqq K
$$

for $N>N_{k}$

for any $m=1, \cdots, 2 k$ and any $p_{i}, 0<p_{i}<2 k, i=1, \ldots, m, \sum_{i=1}^{m} p_{i}=2 k$, $K>0$ is a constant dependent only on $k$. Actually, if $p_{i} \geqq 2$ for $i=1, \cdots, m$, then

$$
\left|\sum_{i_{1}, \cdots, i_{m}=1, \text { different }}^{N} c_{i_{1}}^{p_{1}} \cdots c_{i_{m}}^{p_{m}} \leqq\right| \prod_{j=1}^{m}\left(\sum_{i=1}^{N}\left|c_{i}\right|^{p_{j}}\right) \leqq \max _{1 \leqq i \leqq N}\left|c_{i}\right|^{2(k-m)} .
$$

On the other hand, suppose that some of $p_{i}$ 's are equal to one, say $p_{m}=1$. Then in view of (1.5)

$$
\begin{aligned}
\sum_{i_{1}, \cdots, i_{m}=1, \text { different }}^{N} & c_{i_{1}}^{p_{1}} \cdots c_{i_{1}}^{p_{m}} \\
= & \sum_{i_{1}, \cdots, i_{m-1}=1, \text { different }}^{N} c_{i_{1}}^{p_{1}} \cdots c_{i_{m-1}}^{p_{m-1}}\left(-c_{i_{1}}-\cdots-c_{i_{m-1}}\right)
\end{aligned}
$$

so that we get $m-1$ sums of similar type; each of them sums the products of $(m-1)$ factors. Considering any of these sums, we may have again two cases: 
either all exponents are at least two, so that we are in the first case; or some of them equal one and we may write an equality analogous to (2.8). We continue in this way until after a finite number of steps (in which we decompose the original expression into at most $m$ ! sums) we get only the sums with exponents greater than or equal to two. Actually, the extreme case is the sum of the type

$$
\sum_{i_{1}, i_{2}=1, i_{1} \neq i_{2}}^{N} c_{i_{1}}^{2 k-1} c_{i_{2}}=-\sum_{i_{1}=1}^{N} c_{i_{1}}^{2 k},
$$

so that (2.7) is proved.

Further, using the generalized Cauchy-Schwarz inequality

$$
E\left|\prod_{i=1}^{n} Z_{i}\right| \leqq\left(\prod_{i=1}^{n} E\left|Z_{1}{ }^{n}\right|\right)^{1 / n}, \quad n=2,3, \ldots
$$

we see that

$$
\begin{aligned}
E\left|Y_{i_{1}}^{p_{1}} \cdots Y_{i_{m}}^{p_{m}}\right| & \leqq\left(\prod_{j=1}^{m} E\left|Y_{i_{j}}^{m p_{j}}\right|\right)^{1 / m}<\left(\prod_{j=1}^{m} E\left|Y_{i_{j}}^{2 k p_{j}}\right|\right)^{1 / 2 k} \\
& =\left(\prod_{j=1}^{m} E\left|a_{N}\left(R_{1}\right)-\varphi\left(U_{1}\right)\right|^{2 k p_{j}}\right)^{1 / 2 k}
\end{aligned}
$$

holds for any $m=1, \ldots, 2 k$ and any $p_{i}, 0<p_{i} \leqq 2 k, \sum_{i=1}^{m} p_{i}=2 k$. Finally, the expression

$$
\sum_{m=1}^{2 k} \sum_{1 \leqq p_{1}, \cdots, p_{m} \leqq 2 k, p_{1}+\cdots+p_{m}=2 k} \frac{(2 k) !}{p_{1} ! \cdots p_{m} !}
$$

depends only on $k$.

Now, if $a_{N}(i)=\varphi(i /(N+1)), i=1, \cdots, N$, where $\varphi$ has a bounded derivative we get the inequality

$$
E\left[a_{N}\left(R_{1}\right)-\varphi\left(U_{1}\right)\right]^{2 k p_{j}} \leqq B_{2}(k) E\left[\frac{R_{1}}{N+1}-U_{1}\right]^{2 k p_{j}}
$$

which is varied for $j=1, \ldots, m ; m=1, \ldots, 2 k$.

$U_{1}$ being fixed, $R_{1}$ is the sum of independent zero-one random variables (see (1.2)) so that

$$
E\left(\frac{R_{N 1}}{N+1}-U_{N 1}\right)^{2 k p_{j}} \leqq B_{3}(k) N^{\gamma-k p_{j}} .
$$

(2.6), (2.7), (2.10), (2.11), (2.12) and (2.13) then prove the lemma.

Proof of Theorem 2.1. Since for any $\varepsilon>0$ and any $N$, we have

$$
P\left\{\sigma^{-1} S_{N} \leqq x\right\} \leqq P\left\{\sigma^{-1} T_{N} \leqq x+\varepsilon\right\}+P\left\{\sigma^{-1}\left|S_{N}-T_{N}\right| \geqq \varepsilon\right\}
$$

and analogously

$$
P\left\{\sigma^{-1} S_{N} \leqq x\right\} \geqq P\left\{\sigma^{-1} T_{N} \leqq x-\varepsilon\right\}-P\left\{\sigma^{-1}\left|S_{N}-T_{N}\right| \geqq \varepsilon\right\},
$$

it follows using Lemmas 2.1 and 2.2, that

$$
\sup _{-\infty<x<\infty}\left|F_{N}(x)-\Phi(x)\right| \leqq(\varepsilon \sigma)^{-2 k} B(k) N^{-k}+c_{2} \sum_{i=1}^{N}\left|c_{N i}\right|^{3}+O(\varepsilon)
$$

holds for any $\varepsilon>0$, any $k$ and for $N>N_{k}$.

For $\delta>0$ being fixed, take $k$ such that $2 k+1>1 / 2 \delta \geqq 2 k$ and put $\varepsilon=$ $N^{-\frac{1}{2}(1-1 / 2 k+1)}$. The theorem then follows from (2.13) and from the assumption (1.6). 
3. Rate of convergence for $\Delta \neq 0$. Without loss of generality, we assume that $\Delta>0$. For convenience we shall use the following representation in this section. Let $X_{N i}, i=1, \cdots, N$ be independent and identically distributed rvs each having the $\operatorname{cdf} F(x)$ such that $I(f)<\infty$. Let $R_{N i}^{\Delta}$ be the rank of $X_{N i}+\Delta d_{N i}$, that is

$$
R_{N i}^{\Delta}=\sum_{j=1}^{N} u\left(X_{N i}-X_{N j}+\Delta\left(d_{N i}-d_{N j}\right)\right) .
$$

Consider now the statistics

$$
S_{\Delta N}=\sum_{i=1}^{N} c_{N i} \varphi\left(\frac{R_{N i}^{\Delta}}{N+1}\right) .
$$

The asymptotic distribution of $S_{\Delta N}-S_{0 N}$ was investigated by Jurečková for Wilcoxon scores in (1973a) and for general score function $\varphi$ in (1973b). In the case of general scores function $\varphi$, it was assumed that the $\varphi$ function has the four bounded derivatives in $(0,1)$.

Suppose now that the vectors $\left(c_{N 1}, \cdots, c_{N N}\right)$ and $\left(d_{N 1}, \cdots, d_{N N}\right)$ satisfy (1.5), (1.6) and the following:

$$
\begin{array}{cl}
\lim _{N \rightarrow \infty} \sum_{i=1}^{N} c_{N i} d_{N i}=a^{2}, & 0<a^{2}<\infty, \\
\lim _{N \rightarrow \infty}\left[\max _{1 \leqq i \leqq N}\left(c_{N i}^{2} d_{N i}^{2}\right)\left(\sum_{i=1}^{N} c_{N i}^{2} d_{N i}^{2}\right)^{-1}\right]=0, &
\end{array}
$$

and

$$
\lim _{N \rightarrow \infty}\left[N^{-1}\left(\sum_{i=1}^{N} c_{N i} d_{N i}\right)^{2}\left(\sum_{i=1}^{N} c_{N i}^{2} d_{N i}^{2}\right)^{-1}\right]=\gamma \geqq 0 .
$$

Then, [cf. Jurečková (1973b)] for $\varphi$ having four bounded derivatives in $(0,1)$, the asymptotic distribution of

$$
A_{N}{ }^{-1}\left(S_{\Delta N}-S_{0 N}-\Delta a_{N}-\Delta^{2} b_{N}\right)
$$

is $\eta\left(0, \Delta^{2} \rho^{2}\right)$ where

$$
\begin{gathered}
A_{N}{ }^{2}=\sum_{i=1}^{N} c_{N i}^{2} d_{N i}^{2}+3 N^{-1}\left(\sum_{i=1}^{N} c_{N i} d_{N i}\right)^{2} \\
a_{N}=\sum_{i=1}^{N} c_{N i} d_{N i} \int \varphi^{\prime}(F(x)) f^{2}(x) d x=\sum_{i=1}^{N} c_{N i} d_{N i} \int_{0}^{1} \varphi(t) \varphi(t, f) d t \\
b_{N}=\frac{1}{2} \sum_{i=1}^{N} c_{N i} d_{N i}^{2} \int \varphi^{\prime \prime}(F(x)) f^{3}(x) d x
\end{gathered}
$$

and

$$
\begin{aligned}
\rho^{2}=\int & {\left[\varphi^{\prime}(F(x))\right]^{2} f^{3}(x) d x-\left(\int\left[\varphi^{\prime}(F(x))\right]^{2} f^{2}(x) d x\right)^{2}+2 \gamma(1+3 \gamma)^{-1} } \\
& \times\left[\iint_{x<y} F(x)(1-F(y)) \varphi^{\prime \prime}(F(x)) \varphi^{\prime \prime}(F(y)) f^{2}(x) f^{2}(y) d x d y\right. \\
& +\iint_{x<y} \varphi^{\prime}(F(x)) \varphi^{\prime \prime}(F(y)) f^{2}(x) f^{2}(y) d x d y \\
& -\int \varphi^{\prime}(F(x)) f(x) d x \cdot \int \varphi^{\prime \prime}(F(x)) F(x) f^{2}(x) d x .
\end{aligned}
$$

Let $F_{N \Delta}$ denote the cdf of $\sigma^{-1}\left(S_{\Delta N}-\Delta a_{N}\right)$. Then we have the following theorem.

Theorem 3.1. Suppose that $c_{N i}, d_{N i}, i=1, \ldots, N$ satisfy (1.5), (1.6), (3.1)(3.3) and that the score-generating function has four bounded derivatives on $(0,1)$. 
Then

$$
\sup _{x}\left|F_{N \Delta}(x)-\Phi(x)\right|=O\left(N^{-\frac{1}{2}+\delta}\right)
$$

holds for any $\delta>0$ and any fixed $\Delta$.

Proof. We may write for any $\varepsilon>0$ and for any $x$

$$
\begin{aligned}
& P\left\{\sigma^{-1}\left(S_{\Delta N}-\Delta a_{N}-\Delta^{2} b_{N}\right) \leqq x\right\} \\
& \leqq \\
& \quad+\left\{\sigma^{-1} S_{0 N} \leqq x+\varepsilon\right\} \\
& \quad+P\left\{\sigma^{-1}\left|S_{\Delta N}-S_{0 N}-\Delta a_{N}-\Delta^{2} b_{N}\right| \geqq \varepsilon\right\}
\end{aligned}
$$

and analogously

$$
\begin{aligned}
P\left\{\sigma^{-1}\left(S_{\Delta N}-\Delta a_{N}-\Delta^{2} b_{N}\right) \leqq x\right\} & \\
& \geqq P\left\{\sigma^{-1} S_{0 N} \leqq x-\varepsilon\right\}-P\left\{\sigma^{-1}\left|S_{\Delta N}-S_{0 N}-\Delta a_{N}-\Delta^{2} b_{N}\right| \geqq \varepsilon\right\} .
\end{aligned}
$$

Then by Theorem 2.1,

$$
\begin{aligned}
& \sup _{x}\left|F_{N \Delta}\left(x+\sigma^{-1} \Delta^{2} b_{N}\right)-\Phi(x)\right| \\
& \leqq C \cdot \varepsilon+P\left\{\sigma^{-1}\left|S_{\Delta N}-S_{0 N}-\Delta a_{N}-\Delta^{2} b_{N}\right| \geqq \varepsilon\right\} \\
& \quad+A(\delta) N^{-\frac{1}{2}+\delta}
\end{aligned}
$$

holds for any $\delta>0$ and $N>N_{\tilde{\delta}}$.

Let us consider the third member of the right-hand side of (3.11). We shall use the following theorem:

TheORem 3.2 (Petrov). Let $H(x)$ be any cdf and $\Phi(x)$ cdf of the normal $(0,1)$ distribution.

\section{Let}

$$
\nu=\sup _{-\infty<x<\infty}|H(x)-\Phi(x)|
$$

and let $M_{p}$ denote the set of distribution functions possessing the finite absolute moment of order $p>0$. Then, if $0<\nu \leqq e^{-\frac{1}{2}}$ and $H(x) \in M_{p}$, there exists a constant $C_{p}$ depending on $p$ only such that

$$
|H(x)-\Phi(x)| \leqq \frac{C_{p} \nu\left(\log \frac{1}{\nu}\right)^{p / 2}+\lambda_{p}}{1+|x|^{p}}
$$

holds for all real $x$; here

$$
\lambda_{p}=\left.\left|\int\right| x\right|^{p} d H(x)-\int|x|^{p} d \Phi(x) \mid .
$$

For the proof, see Petrov (1972).

Let us denote by $G_{N \Delta}$ the cdf of $\Delta^{-1} A_{N}{ }^{-1} \rho^{-1}\left(S_{\Delta N}-S_{0 N}-\Delta a_{N}-\Delta^{2} b_{N}\right)$. On account of the boundedness of $\varphi, G_{N \Delta}$ has finite absolute moments of any order for any fixed $N$ and any fixed $\Delta$. On the other hand, it follows from Theorem 2.1 of [6] (see (3.1)-(3.8) of the present paper) that $\lim _{N \rightarrow \infty} \sup _{x} \mid G_{N \Delta}(x)-$ $\Phi(x) \mid=0$ for any fixed $\Delta$ and that for $N>N_{\Delta}$

$$
\sup _{x}\left|G_{N \Delta}(x)-\Phi(x)\right|<e^{-\frac{1}{2}} \text {. }
$$


The assumptions of Theorem 3.2 are satisfied for any $p=k=1,2, \ldots$, so that there exists a constant $C_{k}{ }^{*}$ to any $k$ such that

$$
\left|G_{N \Delta}(x)-\Phi(x)\right| \leqq C_{k}^{*}\left(1+|x|^{k}\right)^{-1}
$$

holds for all $x \in(-\infty, \infty)$.

We have

$$
P\left\{\sigma^{-1}\left|S_{\Delta N}-S_{0 N}-\Delta a_{N}-\Delta^{2} b_{N}\right| \geqq \varepsilon\right\}=2\left[1-G_{N \Delta}\left(\Delta^{-1} \rho^{-1} \sigma A_{N}{ }^{-1} \varepsilon\right)\right]
$$

so that (3.13) implies that

$$
\begin{aligned}
P\left\{\sigma^{-1} \mid S_{\Delta N}-\right. & \left.S_{0 N}-\Delta a_{N}-\Delta^{2} b_{N} \mid \geqq \varepsilon\right\} \\
& \leqq 2\left[1-\Phi\left(\Delta^{-1} \rho^{-1} \sigma A_{N}^{-1} \varepsilon\right)\right]+2 C_{k} *\left[1+\left(\Delta^{-1} \rho^{-1} \sigma\right)^{k} A_{N}{ }^{-k} \varepsilon^{k}\right]^{-1}
\end{aligned}
$$

holds for any $\varepsilon>0$, any $k=1,2, \ldots$ and for $N>N_{\Delta}$.

Let us fix $\delta, \delta>0$ and put $\varepsilon=A_{N} \cdot N^{\delta / 2}$. Then in view of (3.15) and Lemma 2, Chapter VII of Feller (1957) we have that for any $N>N_{\Delta}$ and sufficiently large $k$

$$
\sup \left|F_{N \Delta}\left(x+\sigma^{-1} \Delta^{2} b_{N}\right)-\Phi(x)\right| \leqq C_{\delta}^{\prime \prime} N^{-\frac{1}{2}+\delta}+O\left(N^{-1+2 \delta}\right)
$$

Thus

$$
\begin{aligned}
& \sup _{-\infty<x<\infty}\left|F_{N \Delta}(x)-\Phi(x)\right| \\
& \leqq \sup _{x}\left|F_{N \Delta}(x)-\Phi\left(x+\sigma^{-1} \Delta^{2} b_{N}\right)\right| \\
& +\sup _{x}\left|\Phi\left(x+\sigma^{-1} \Delta^{2} b_{N}\right)-\Phi(x)\right| \\
& \leqq \sup _{x}\left|F_{N \Delta}\left(x-\sigma^{-1} \Delta^{2} b_{N}\right)-\Phi(x)\right|+K \cdot \sigma^{-1} \Delta^{2} b_{N} .
\end{aligned}
$$

(3.16) and (3.17) together with assumption (1.5) complete the proof of the Theorem.

Acknowledgment. It is a pleasure to express our appreciation to Dr. Søren Johansen and Martin Jacobsen for some helpful discussions, and to the referee for some very valuable comments. We would also like to thank the referee for bringing to our attention a paper of Bickel (1974) where he established that a Berry-Esseén bound of order $N^{-\frac{1}{2}}$ was valid for the Wilcoxon statistic. This suggests that the bound in the present paper may be improved upon. The reader is also referred to Vizková, (1974) for a related problem.

\section{REFERENCES}

[1] Bickel, P. J. (1974). Edgeworth expansions in nonparametric statistics. Ann. Statist. 2 1-20.

[2] Capon, J. (1961). Asymptotic efficiency of certain locally most powerful rank tests. Ann. Math. Statist. 32 88-100.

[3] Feller, W. (1957). An Introduction to Probability Theory and its Applications. Wiley, New York.

[4] Hájek, J. (1962). Asymptotically most powerful rank order tests. Ann. Math. Statist. 33 $1124-1147$.

[5] HÁjeK, J. and SidÁc, Z. (1967). Theory of Rank Tests. Academic Press, New York.

[6] JuReč́ovÁ, J. (1973 a). Central limit theorem for Wilcoxon rank statistics process. Ann. Statist. 1 1046-1060. 
[7] JurečKovÁ, J. (1973 b). Central limit theorem for rank statistics process. (Submitted).

[8] Petrov, V. V. (1972). The Sums of Independent Random Variables (in Russian). Nauka, Moscow.

[9] Vízková, Z. (1974). Local limit theorems for rank statistics. Ph. D. dissertation, Charles Univ., Prague.

JANA JUReČKovÁ

Charles University

Department of Statistics

SOKOLOVSKA UL. 83

Prague 8, Czechoslovakia
Madan L. Puri

InStitut fur Mathematische Statistick UND WIRTSCHAFTSMATIK

UNIVERSITAT GOTTINGEN

34 GotTingen

Lotzestrabe 13, West Germany 